\title{
Equations of Motion from Field Equations and a Gauge-invariant Variational Principle for the Motion of Charged Particles
}

\author{
Dariusz Chruściński* \\ Institute of Physics, Nicholas Copernicus University \\ ul. Grudziạdzka 5/7, 87-100 Toruń, Poland \\ and \\ Jerzy Kijowski ${ }^{\dagger}$ \\ Centrum Fizyki Teoretycznej PAN \\ Aleja Lotników 32/46, 02-668 Warsaw, Poland
}

\begin{abstract}
New, gauge-independent, second order Lagrangian for the motion of classical, charged test particles is proposed. It differs from the standard, gauge-dependent, first order Lagrangian by boundary terms only. A new method of deriving equations of motion from field equations is developed. When applied to classical electrodynamics, this method enables us to obtain unambiguously the above, second order Lagrangian from the general energy-momentum conservation principle.
\end{abstract}

\section{Introduction}

The motion of classical, charged test particles, in the classical Maxwell field is derived usually from the gauge-dependent Lagrangian function

$$
L=L_{\text {particle }}+L_{i n t}=-\sqrt{1-\mathbf{v}^{2}}\left(m-e u^{\mu} A_{\mu}(t, \mathbf{q})\right),
$$

where $u^{\mu}$ denotes the (normalized) four-velocity vector

$$
\left(u^{\mu}\right)=\left(u^{0}, u^{k}\right):=\frac{1}{\sqrt{1-\mathbf{v}^{2}}}\left(1, v^{k}\right),
$$

and $v^{k}:=\dot{q}^{k}$ (we use the Heaviside-Lorentz system of units with the velocity of light $c=1$ ).

Since the Lorentz force $e u^{\mu} f_{\mu \nu}$ derived from this Lagrangian is perfectly gauge invariant, it is not clear, why we have to use the gauge-dependent interaction term $e u^{\mu} A_{\mu}$, with no direct physical interpretation. Moreover, in this approach equations of motion are not uniquely implied by field equations. As an example, non-linear forces of the type $u_{\mu} f^{\mu \lambda} u^{\kappa} \nabla_{\lambda} f_{\kappa \nu}$ cannot be a priori excluded.

* e-mail: darch@phys.uni.torun.pl

$\dagger$ e-mail: kijowski@cft.edu.pl 
In the present paper we show that the same Lorentz force may be derived from a gaugeinvariant, second order Lagrangian $\mathcal{L}$ :

$$
\mathcal{L}=L_{\text {particle }}+\mathcal{L}_{\text {int }}=-\sqrt{1-\mathbf{v}^{2}}\left(m-a^{\mu} u^{\nu} M_{\mu \nu}^{\text {int }}(t, \mathbf{q}, \mathbf{v})\right),
$$

where $a^{\mu}:=u^{\nu} \nabla_{\nu} u^{\mu}$ is the particle's acceleration. The skew-symmetric tensor $M_{\mu \nu}^{\text {int }}(t, \mathbf{q}, \mathbf{v})$ is equal to the amount of the angular-momentum of the field, which is acquired by our physical system, when the (boosted) Coulomb field $\mathbf{f}_{\mu \nu}^{(y, u)}$, accompanying the particle moving with constant velocity $u$ through the space-time point $y=(t, \mathbf{q})$, is added to the background (external) field. More precisely: the total energy-momentum tensor corresponding to the sum of the background field $f_{\mu \nu}$ and the above Coulomb field $\mathbf{f}_{\mu \nu}^{(y, u)}$ decomposes in a natural way into a sum of 1) terms quadratic in $\left.f_{\mu \nu}, 2\right)$ terms quadratic in $\mathbf{f}_{\mu \nu}^{(y, u)}$ and 3) mixed terms. The quantity $M_{\mu \nu}^{\text {int }}$ is equal to this part of the total angular-momentum $M_{\mu \nu}$, which we obtain integrating only the mixed terms of the energy-momentum tensor. It is proportional to the particle's electric charge $e$ contained in the particle's Coulomb field. The interaction Lagrangian $\mathcal{L}_{\text {int }}$ can thus be expressed in terms of the following quantity

$$
Q_{\mu}:=\frac{1}{e} u^{\nu} M_{\mu \nu}^{i n t},
$$

which is obviously orthogonal to the four-velocity $u^{\mu}$. Therefore, it has only three independent components. In the particle's rest frame we have $Q_{0}=0$ and the three-vector $e Q_{k}$ equals to the amount of the static moment acquired by the system when the particle's own field is added to the background (external) field. Hence, we have from (3) and (4):

$$
\mathcal{L}_{\text {int }}=\sqrt{1-\mathbf{v}^{2}} e a^{\mu} Q_{\mu}(t, \mathbf{q}, \mathbf{v}) .
$$

We prove in Section 2 that, for a given external field $f_{\mu \nu}$, the new interaction Lagrangian (5) differs from the old one by (gauge-dependent) boundary corrections only. Hence, both Lagrangians generate the same physical theory (although the new Lagrangian is of second differential order, its dependence upon the second derivatives is linear; this implies that the corresponding Euler - Lagrange equations of motion are of the second order). The relation between $L$ and $\mathcal{L}$ is, therefore, analogous to the one well known in General Relativity: the gauge-invariant, second order Hilbert Lagrangian for Einstein equations may be obtained starting from the first order, gauge-dependent Lagrangian and supplementing it by an appropriate boundary term.

At this point, our result can be summarized as follows: a physical interpretation of the interaction Lagrangian $e u^{\mu} A_{\mu}$ has been found. Up to boundary terms, it is equal to the interactionangular-momentum $a^{\mu} u^{\nu} M_{\mu \nu}^{i n t}$. The question arises whether such an identity is a pure coincidence or is a result of a universal law of physics.

In the second part of the paper (Sections $3-5$ ) we try to convince the reader that the second conjecture is correct. In fact, we propose a new method of deriving equations of motion from field equations. The method is based on an analysis of the geometric structure of generators of the Poincaré group, related with any special-relativistic, lagrangian field theory. This analysis leads us to a simple theorem, which we call "variational principle for an observer" (see Section 3). Applying this observation to the specific case of classical electrodynamics, we show how to derive, in principle, equations of motion from field equations. This derivation is based on a following heuristic idea: a point-like particle has to be understood as an approximation of an extended particle, i. e. of an exact, stable, soliton-like solution of a hypothetical theory of matter 
fields interacting with electromagnetic field. To prove that the Maxwell theory (describing the free field outside of the "soliton's strong-field-core") implies equations of motion for the solitons themselves, we need several qualitative assumptions of heuristic nature, about their stability. Under these assumptions the gauge-invariant Lagrangian describing the motion of point particles is unambiguously derived from the general invariance principles of the theory.

The mathematical status of the above derivation is, therefore, similar to the Einstein - Infeld - Hoffmann derivation of equations of motion from field equations in General Relativity. It does not depend upon a particular model which we take for the description of the gravitating body under consideration (e. g. a hydrodynamical or an elastomechanical model). The derivation is valid for any stable body and enables us to describe (in a good approximation) its motion in a model-independent way, as a geodesic motion of a point-particle. Hence, even if we have at the moment no realistic mathematical theory describing the interior of a star and fulfilling all the necessary assumptions, we can expect that the above equations of motion are valid for relatively stable objects. The present paper shows that a similar argumentation is possible also in electrodynamics: Lorentz force acting on test particles does not need to be postulated as an independent physical law, but is implied by the geometry of Maxwell field, provided one accepts the existence of the hypothetical fundamental theory of matter fields, admitting sufficiently stable soliton-like solutions.

The above result follows immediately from the consistent theory of interacting particles and fields (cf. [1], [2]), called Electrodynamics of Moving Particles. All the formulae of the present paper can be derived directly from the above theory in the test particle limit (i.e. $m \rightarrow 0, e \rightarrow 0$ with the ratio $e / m$ being fixed). The present paper, however, does not rely on this, much more general, context. The consistent theory of test particles is constructed independently.

The paper is organized as follows. Section 2 contains a direct proof that our new Lagrangian differs from the standard, gauge-dependent one by boundary terms only. Section 3 contains our basic geometric observation concerning any relativistic, Lagrangian field theory, which makes our approach possible. Using it, we give in Sections 1 and 5 the derivation of our variational principle. In particular, the renormalization procedure defined in Section 5 depends upon the stability assumptions (which may be relatively difficult to check for any specific mathematical model). Accepting these assumptions on a heuristic level we obtain our theory of point particles uniquely, as a necessary consequence of energy-momentum conservation.

In a forthcoming paper we are going to present the gauge-invariant Hamiltonian structure of the above theory.

\section{Equivalence between the two variational principles}

The easiest way to prove the equivalence consists in rewriting the field $f_{\mu \nu}$ in terms of the electric and the magnetic induction, using a special accelerated reference system, adapted to the particle's trajectory $\zeta$, which we define in the sequel. The system will be also very useful to formulate our "variational principle for an observer" in the next Section.

We begin with any laboratory coordinate system $\left(y^{\mu}\right)=\left(y^{0}, y^{k}\right)$ in Minkowski space-time $M$ and parameterize the trajectory $\zeta$ with the laboratory time $t=y^{0}$. Let $y^{k}=\mathbf{q}(t)$ be the corresponding coordinate description of $\zeta$. At each point of the trajectory we choose an orthonormal tetrad $\left(\mathbf{e}_{\mu}\right)$, such that its element $\mathbf{e}_{0}$ is tangent to $\zeta$, i. e. is equal to the four-velocity vector $u$. Take now the unique boost transformation relating the laboratory time axis $\partial / \partial y^{0}$ 
with the observer's time axis $\mathbf{e}_{0}$. We define the vector $\mathbf{e}_{k}$ by transforming the corresponding $\partial / \partial y^{k}-$ axis of the laboratory frame by the same boost.

It is easy to check (cf. [2]) that the above definition implies the following, explicit formula:

$$
\mathbf{e}_{k}=\frac{v_{k}}{\sqrt{1-\mathbf{v}^{2}(t)}} \frac{\partial}{\partial y^{0}}+\left(\delta_{k}^{l}+\varphi\left(\mathbf{v}^{2}\right) v^{l} v_{k}\right) \frac{\partial}{\partial y^{l}},
$$

where we denote $\varphi(z):=\frac{1}{z}\left(\frac{1}{\sqrt{1-z}}-1\right)=\frac{1}{\sqrt{1-z}(1+\sqrt{1-z})}$.

Finally, we parameterize space-time points by four coordinates $\left(t, x^{k}\right)$ :

$$
y(t, x):=(t, \mathbf{q}(t))+x^{k} \mathbf{e}_{k}(t) .
$$

Using (6) we obtain the following relation between our curvilinear parameters $\left(t, x^{k}\right)$ and the laboratory (Lorentzian) coordinates $\left(y^{\mu}\right)$ :

$$
\begin{aligned}
y^{0}\left(t, x^{l}\right) & :=t+\frac{1}{\sqrt{1-\mathbf{v}^{2}(t)}} x^{l} v_{l}(t), \\
y^{k}\left(t, x^{l}\right) & :=q^{k}(t)+\left(\delta_{l}^{k}+\varphi\left(\mathbf{v}^{2}\right) v^{k} v_{l}\right) x^{l} .
\end{aligned}
$$

The above formula may be used as a starting point of the entire proof. To rewrite field equations with respect to this system we need to calculate the components of the flat Minkowskian metric in our new coordinates. We see from (7]) that, for a given value $t$, parameters $\left(x^{k}\right)$ are cartesian coordinates on the 3 -dimensional hyperplane $\Sigma_{t}$, orthogonal to $\zeta$ at the point $(t, \mathbf{q}(t))$. Hence, we get $g_{k l}=\delta_{k l}$ for the space-space components of the metric.

The remaining information about the metric is carried by the lapse function and the shift vector, which again may be easily calculated from formula (8):

$$
\begin{aligned}
N & =\frac{1}{\sqrt{-g^{00}}}=\sqrt{1-\mathbf{v}^{2}}\left(1+a_{i} x^{i}\right), \\
N_{m} & =g_{0 m}=\sqrt{1-\mathbf{v}^{2}} \epsilon_{m k l} \omega^{k} x^{l} .
\end{aligned}
$$

Here, by $a^{i}$ we denote the rest-frame components of the particle's acceleration. They are given by formula:

$$
\frac{d}{d \tau} \mathbf{e}_{0}=\frac{d}{d \tau} u=a^{i} \mathbf{e}_{i}
$$

where $\tau$ is the proper time along $\zeta$. A straighforward calculation gives us (formulae (2) and (66)) the following value:

$$
a^{i}=\frac{1}{1-\mathbf{v}^{2}}\left(\delta_{k}^{i}+\varphi\left(\mathbf{v}^{2}\right) v^{i} v_{k}\right) \dot{v}^{k}
$$

where $\dot{v}^{k}$ is the acceleration in the laboratory frame.

Moreover, at each point of $\zeta$ we define the rotation vector $\left(\omega^{j}\right)$ of the tetrad by the following formula:

$$
\frac{d}{d \tau} \mathbf{e}_{i}=a_{i} \mathbf{e}_{0}-\epsilon_{i j}{ }^{k} \omega^{j} \mathbf{e}_{k}
$$

Again, straighforward calculation leads to the following expression:

$$
\omega_{m}=\frac{1}{\sqrt{1-\mathbf{v}^{2}}} \varphi\left(\mathbf{v}^{2}\right) v^{k} \dot{v}^{l} \epsilon_{k l m}
$$


The transformation (8) is not invertible. Coordinates $\left(x^{k}\right)$ are regular parameters on each $\Sigma_{t}$. But hyperplanes $\Sigma_{t}$ corresponding to different values of $t$ may intersect. Hence, the same physical event may correspond to different values of coordinates $\left(t, x^{k}\right)$.

Nevertheless, we may describe the free Maxwell field in terms of our parameters $\left(t, x^{k}\right)$. In particular, we have:

$$
\dot{A}_{k}-\partial_{k} A_{0}=: f_{0 k}=-N D_{k}+\epsilon_{m k l} N^{m} B^{l},
$$

where $D^{k}$ and $B^{k}$ are the electric and the magnetic field on each $\Sigma_{t}, N$ and $N^{m}$ are given by (9) and (10) (for the description of the Maxwell field with respect to an accelerated reference system see e. g. [3]).

Let us multiply (15) by $\frac{x^{k}}{r^{3}}$ and integrate this scalar product over $\Sigma_{t}$ with respect to the Lebesgue measure $d x^{3}$. We first integrate over the exterior of the sphere $S\left(r_{0}\right)$. Observe, that in this region the following identity holds:

$$
\frac{x^{k}}{r^{3}} \partial_{k} A_{0}=\partial_{k} \frac{x^{k} A_{0}}{r^{3}} .
$$

Moreover, we have:

$$
\frac{x^{k}}{r^{3}} \epsilon_{m k l} N^{m} B^{l}=-\sqrt{1-\mathbf{v}^{2}} \partial_{l}\left(B^{l} \frac{\omega_{k} x^{k}}{r}\right) .
$$

Hence, after integration, we obtain in the limit $r_{0} \rightarrow 0$ :

$$
\int_{\Sigma_{t}} \frac{x^{k}}{r^{3}} \dot{A}_{k} d^{3} x+4 \pi A_{0}(t, 0)=-\int_{\Sigma_{t}} \frac{x^{k}}{r^{3}} N D_{k} d^{3} x
$$

$\left(A_{0}(t, 0)\right.$ is the only surface term which survives in the limit, due to the standard asymptotic behaviour of the field). Observe that the constant part of the lapse function (9) does not produce any contribution to the right-hand side of the above formula, because the flux of the field $D_{k}$ through any sphere $S(r)$ vanishes due to the Gauss law. Hence, we may replace " $N$ " by " $\sqrt{1-\mathbf{v}^{2}} a_{i} x^{i}$ " under the integral and obtain

$$
\begin{aligned}
\int_{\Sigma_{t}} \frac{x^{k}}{r^{3}} N D_{k} d^{3} x & =\frac{4 \pi}{e} \sqrt{1-\mathbf{v}^{2}} a_{k} \int_{\Sigma_{t}} x^{k} \mathbf{D}^{n} D_{n} d^{3} x \\
& =-\frac{4 \pi}{e} a^{k} M_{k 0}^{i n t}=-\frac{4 \pi}{e} a^{\mu} M_{\mu 0}^{i n t},
\end{aligned}
$$

where

$$
\mathbf{D}^{n}:=\frac{e}{4 \pi} \frac{x^{n}}{r^{3}}
$$

is the Coulomb field on $\Sigma_{t}$, corresponding to the charge $e$.

The lower index " 0 " in our particular system comes from the vector $\frac{\partial}{\partial t}$ which is proportional to the particle's velocity $u^{\mu}$, where the proportionality coefficient $\sqrt{1-\mathbf{v}^{2}}$ is due to the ratio between the proper time and the laboratory time on the trajectory. This means that $A_{0}(t, 0)$, calculated in our particular coordinate system, is equal to $\sqrt{1-\mathbf{v}^{2}} u^{\mu} A_{\mu}(t, \mathbf{q}(t))$ in any other coordinate system. The same is true for $M_{\mu 0}^{i n t}$. We have, therefore:

$$
\frac{e}{4 \pi} \int_{\Sigma_{t}} \frac{x^{k}}{r^{3}} \dot{A}_{k} d^{3} x+\sqrt{1-\mathbf{v}^{2}} e u^{\mu} A_{\mu}(t, \mathbf{q}(t))=\sqrt{1-\mathbf{v}^{2}} a^{\mu} u^{\nu} M_{\mu \nu}^{i n t}
$$


Integrating this identity over a time interval $\left[t_{1}, t_{2}\right]$ we finally obtain

$$
\int_{t_{1}}^{t_{2}} \mathcal{L}_{\text {int }}=\int_{t_{1}}^{t_{2}} L_{i n t}-\frac{e}{4 \pi}\left(\int_{\Sigma_{t_{2}}} \frac{x^{k}}{r^{3}} A_{k} d^{3} x-\int_{\Sigma_{t_{1}}} \frac{x^{k}}{r^{3}} A_{k} d^{3} x\right),
$$

i. e. both Lagrangians differ by boundary terms only.

We will see in the sequel that the value of $M_{\mu \nu}^{\text {int }}$ does not depend upon the choice of a particular hypersurface $\Sigma_{t}$ which we have used for integration. Any other $\Sigma$, which intersects the trajectory at the same point and is flat at infinity will give the same result. We conclude that there is always a gauge in which both $\mathcal{L}$ and $L$ coincide, e. g. the gauge in which the monopole part of the radial component $A_{r}:=A_{k} \frac{x^{k}}{r}$ vanishes.

\section{Variational principle for an observer}

Consider any relativistic-invariant, Lagrangian field theory (in this paper we will consider mainly Maxwell electrodynamics, but the construction given in the present Section may be applied to any scalar, spinor, tensor or even more general field theory). Choose any non-inertial observer, moving along a time-like trajectory $\zeta$. We want to describe the field evolution with respect to the observer's rest frame. For this purpose we choose the space-time parameterization defined in the previous Section.

The field evolution with respect to the above non-inertial reference frame is a superposition of the following three transformations:

- time-translation in the direction of the local time-axis of the observer,

- boost in the direction of the acceleration $a^{k}$ of the observer,

- purely spatial $\mathrm{O}(3)$-rotation $\omega^{m}$.

It is, therefore, obvious that the field-theoretical generator of this evolution is equal to

$$
H=\sqrt{1-\mathbf{v}^{2}}\left(\mathcal{E}+a^{k} R_{k}-\omega^{m} S_{m}\right),
$$

where $\mathcal{E}$ is the rest-frame field energy, $R_{k}$ is the rest-frame static moment and $S_{m}$ is the restframe angular momentum. The factor $\sqrt{1-\mathbf{v}^{2}}$ in front of the generator is necessary, because the time $t=x^{0}$, which we used to parameterize the observer's trajectory, is not the proper time along $\zeta$ but the laboratory time. For any point $(t, \mathbf{q}(t)) \in \zeta$ the values of the Poincaré generators $\mathcal{E}, R_{k}$ and $S_{m}$ are given as integrals of appropriate components of the field energymomentum tensor over any space-like Cauchy surface $\Sigma$ which intersects $\zeta$ precisely at $(t, \mathbf{q}(t))$ (due to Noether's theorem, the integrals are independent upon the choice of such a surface). These values are, therefore, equal to the components of the total four-momentum $p_{\mu}$ and the total angular momentum $M_{\mu \nu}$ of the field, calculated in the observer's co-moving frame $\mathbf{e}_{\mu}$, i.e. $\mathcal{E}=-p_{\mu} u^{\mu}, R_{\mu}=-M_{\mu \nu} u^{\nu}$ and $R_{\mu} u^{\mu}=0$.

Given a field configuration, we are going to use the quantity $H$ as a second order Lagrangian for the observer's trajectory. For this purpose let us first choose a "reference trajectory" $\zeta_{0}$. Next, for each point $(t, \mathbf{q}(t)) \in \zeta_{0}$ let us calculate the corresponding "reference values" of the generators $\mathcal{E}(t), R_{k}(t), S_{m}(t)$ and insert them into $H$. Finally, consider the function (23) obtained this way as a Lagrangian depending upon a generic trajectory $\zeta$ via its velocity $v$ and acceleration $\dot{v}$, according to (12) and (14). 


\section{Theorem}

Euler-Lagrange equations derived from the above Lagrangian are automatically satisfied by the trajectory $\zeta=\zeta_{0}$.

This theorem was derived in [2] in a much more general framework. Within this framework, it was an obvious consequence of the invariance of the theory with respect to the choice of an observer. More precisely, the function " $-H$ " was proved to be a Routhian function playing the role of the Lagrangian with respect to the observer's degrees of freedom and the Hamiltonian (with opposite sign) with respect to the field degrees of freedom. For purposes of the present paper we do not need, however, this larger context. The Theorem may be checked by simple inspection: Euler-Lagrange equations derived from the second order Lagrangian (23) are automatically satisfied as a consequence of the field energy-momentum and angular-momentum conservation (this direct proof was also given in [2]).

\section{Adding a test particle to the field}

From now on we limit ourselves to the case of electrodynamics. This means that the field energymomentum and angular-momentum are defined as appropriate integrals of the components of the Maxwell energy-momentum tensor

$$
T_{\nu}^{\mu}=f^{\mu \lambda} f_{\nu \lambda}-\frac{1}{4} \delta_{\nu}^{\mu} f^{\kappa \lambda} f_{\kappa \lambda}
$$

Suppose now that to a given background field $f_{\mu \nu}$ we add a test particle carrying an electric charge $e$. Denote by $\mathbf{f}_{\mu \nu}^{(y, u)}$ the (boosted) Coulomb field accompanying the particle moving with constant four-velocity $u$, which passes through the space-time point $y$. Being bi-linear in fields, the energy-momentum tensor $T^{\text {total }}$ of the total field

$$
f_{\mu \nu}^{\text {total }}:=f_{\mu \nu}+\mathbf{f}_{\mu \nu}^{(y, u)}
$$

may be decomposed into three terms: the energy-momentum tensor of the background field $T^{\text {field }}$, the Coulomb energy-momentum tensor $T^{\text {particle }}$, which is composed of terms quadratic in $\mathbf{f}_{\mu \nu}^{(y, u)}$ and the "interaction tensor" $T^{i n t}$, containing mixed terms:

$$
T^{\text {total }}=T^{\text {field }}+T^{\text {particle }}+T^{\text {int }} .
$$

Unfortunately, $T^{\text {total }}$ does not lead any longer to a globally conserved quantity, as in the case of a relativistic invariant field theory. Indeed, the relativistic invariance has been broken by the choice of the electric current localized on the trajectory. Moreover, due to the Coulomb field's singularity, integrals which would have been necessary to obtain the generator (23) from (26) are ill defined. Hence, techniques introduced in Section 3 cannot be used directly.

For reasons which will be fully explained in the next Section, we replace (23) by the following, well defined, "renormalized" quantity. To obtain this quantity we will integrate first two terms of (26) over any $\Sigma$ which passes through $y=(t, \mathbf{q}(t))$. Integration of $T^{\text {field }}$ (no singularity) and $T^{\text {int }}$ (an $r^{-2}$ - singularity) is possible and gives well defined quantities, which we call respectively $H^{\text {field }}$ ("background field generator") and $H^{\text {int }}$ ("interaction generator"). Because the left-hand side and the first two terms of the right-hand side of (26) have a vanishing divergence (outside of the particle's trajectory), we conclude that also $T^{\text {int }}$ has a vanishing divergence. This implies, that the above integrals are invariant with respect to changes of $\Sigma$, provided the intersection point with the trajectory does not change (see [1] for more details). 
Unfortunately, the Coulomb tensor $T^{\text {particle }}$ has an $r^{-4}$ singularity at $y$ and cannot be integrated. According to the renormalization procedure defined in [2] and sketched briefly in the next Section, we replace its integrals by the corresponding components of the total fourmomentum of the particle: $p_{\lambda}^{\text {particle }}=m u_{\lambda}$ and the total angular momentum: $M_{\mu \nu}^{\text {particle }}=0$. We define, therefore, the renormalized particle generator as follows:

$$
H_{\text {ren }}^{\text {particle }}=m \sqrt{1-\mathbf{v}^{2}} .
$$

Consequently, we define the total (already renormalized) generator as a sum of three terms

$$
H_{\text {ren }}^{\text {total }}=H^{\text {field }}+H^{\text {int }}+H_{\text {ren }}^{\text {particle }},
$$

where the first term is quadratic and the second term is linear with respect to the background field $f_{\mu \nu}$.

Let us observe that the only non-vanishing term in $H^{\text {int }}$ comes from the static moment term $R$ in (23), because the mixed terms in both the energy $\mathcal{E}$ and the angular momentum $S$ vanish when intergated over any $\Sigma$. The easiest way to prove this fact consists in choosing the hypersurface $\Sigma_{t}$ which is orthogonal to the velocity $u$ at $(t, \mathbf{q}(t))$, i.e. the rest-frame surface (our integrals do not depend upon the choice of a hypersurface). On this surface, the Coulomb

field $\mathbf{f}_{\mu \nu}^{(y, u)}$ is spherically symmetric and carries, therefore, only the monopole component. On the other hand, the monopole component of the background field $f_{\mu \nu}$ vanishes as a consequence of the homogeneous Maxwell equations (no charges!). The mixed term in the energy integral is, therefore, a product of a monopole-free functions and the pure monopole. Hence, it vanishes after integration. A similar argument applies to the angular momentum $S$.

Finally, we have defined

$$
H_{r e n}^{\text {total }}=H^{\text {field }}-\sqrt{1-\mathbf{v}^{2}} a^{\mu} u^{\nu} M_{\mu \nu}^{i n t}(t, \mathbf{q}, \mathbf{v})+\sqrt{1-\mathbf{v}^{2}} m,
$$

where the interaction term is defined as the following integral

$$
M_{\mu \nu}^{i n t}(y):=\int_{\Sigma}\left\{\left(x_{\mu}-y_{\mu}\right) T_{\nu \lambda}^{i n t}(x)-\left(x_{\nu}-y_{\nu}\right) T_{\mu \lambda}^{i n t}(x)\right\} d \Sigma^{\lambda}(x),
$$

and $\Sigma$ is any hypersurface which intersects the trajectory at the point $y=(t, \mathbf{q}(t))$.

In particular, using the particle's rest-frame and integrating over the rest-frame hypersurface $\Sigma_{t}$ we obtain formula (19) for $M_{k 0}^{i n t}$.

As we have already mentioned, the quantity $H_{r e n}^{\text {total }}$ defined this way, cannot be directly used in the framework of pure electrodynamics, as the "observer's Lagrangian". In the next Section we will show, however, that this quantity provides a good approximation of the total generator $H$ within a more general framework. In this framework particles are no longer point-like, but are extended objects, described by matter fields interacting with electrodynamics. We will conclude, that within this approximation our "renormalized generator" may be used to derive an "approximative trajectory" of such extended objects.

\section{Renormalization. Derivation of equations of motion from field equations}

According to the approach developed in [1] and [2] we treat the moving particle as a solution of a hypothetical "fundamental theory of matter fields interacting with electromagnetic field". We 
assume that such a theory is a relativistic, Lagrangian (possibly highly non-linear) field theory. Moreover, we assume linear Maxwell theory as a limiting case of the above theory, corresponding to sufficiently weak electromagnetic fields and vanishing matter fields.

We will suppose that the particle, whose interaction with the electromagnetic field we are going to analyze, is a global solution of the above field theory, having following qualitative properties:

1. it contains a tiny "strong field region", concentrated in the vicinity of a time-like trajectory $\zeta$, which we may call an approximate trajectory of the extended particle,

2. outside of this strong field region the matter fields vanish (or almost vanish in the sense, that the following approximation remains valid) and the electromagnetic field is sufficiently weak to be described by linear Maxwell equations.

To be more precise, we imagine the "particle at rest" as a stable, static, soliton-like solution of our hypothetical "super theory". The solution is characterized by two parameters: its total charge $e$ and its total energy $m$. The energy is not concentrated within the interior of the particle but contains also the part of the energy carried by its "Coulomb tail". This means that $m$ is an already renormalized mass, (or dressed mass), including the energy of the field surrounding the particle. Within this framework questions like "how big the bare mass of the particle is and which part of the mass is provided by the purely electromagnetic energy?" are meaningless. In the strong field region (i. e. inside the particle) the energy density may be highly non-linear and there is probably no way to divide it consistently into two such components.

Due to relativistic invariance of the theory, there is a 6 parameter family of the "uniformly moving particle" solutions obtained from our soliton via Poincaré transformations.

Now, an arbitrarily moving particle is understood as a "perturbed soliton". This means that it is again an exact solution of the same "super theory", with its strong-field-region concentrated in the vicinity of a time-like world line $\zeta$, which is no longer a straight line, as it was for "uniformly moving particles". Let us choose an observer who follows this "approximate trajectory" $\zeta$. We know that he automatically satisfies the Euler-Lagrange equations derived from the second order Lagrangian (23), where $\mathcal{E}, R^{k}$ and $S^{m}$ are the quantities calculated for the complete non-linear theory.

Suppose now, that the particle may be treated as a test particle. This means, that the total field outside of the particle does not differ considerably from a background field $f$, satisfying homogeneous Maxwell equations. Using this hypothesis we may approximate, for each point $(t, \mathbf{q}(t))$, the exact value of $(23)$ by the value of (29). Indeed, we may decompose the total energy-momentum tensor of the complete non-linear field as follows:

$$
T_{\lambda}^{\mu}=\left(T_{\lambda}^{\mu}-\mathbf{T}_{\lambda}^{\mu}\right)+\mathbf{T}_{\lambda}^{\mu} .
$$

Here, by $\mathbf{T}_{\lambda}^{\mu}$ we denote the total energy-momentum of the "super theory", corresponding to the "uniformly moving particle" solution, which matches on $\Sigma$ the position and the velocity of our particle. Stability of the soliton means that the the "moving particle solution" does not differ considerably from the "uniformly moving particle solution" inside of the strong field region (i. e. inside the particles). Hence, the contribution of the first term $(T-\mathbf{T})$ to the integrals $\mathcal{E}$, $R^{k}$ and $S^{m}$ may be neglected "inside the particle", i. e. we may replace it under integration by the purely Maxwellian quantity

$$
\left(T^{\text {total }}-T^{\text {particle }}\right)=T^{\text {field }}+T^{\text {int }} .
$$


As a result, we obtain the first two terms of (29). On the other hand, integrating the last term $\mathbf{T}$ in (31) we obtain without any approximation the corresponding value of the four-momentum of the particle. This way we reproduce the last term of (29).

Replacing (23) by its approximate value (29) and using the Theorem we conclude that the trajectory $\zeta$ has to fulfill Euler-Lagrange equations derived from (29). Applying the Theorem to the linear Maxwell theory we conclude, that the term $H^{\text {field }}$ produces Euler-Lagrange equations which are automatically fulfilled by $\zeta$. Hence, we may drop out this term, leaving only the remaining two terms. They finally give us our formula (3) for the Lagrangian of the test particle (the sign has to be changed because as a Lagrangian we should have taken " $-H$ " instead of " $H$ " - see remark at the end of Section 3).

\section{References}

[1] J. Kijowski, Electrodynamics of Moving Particles, Gen. Rel. and Grav. 26 (1994) 167

J. Kijowski, On Electrodynamical Self-interaction, Acta Phys. Polon. A85 (1994) 771

[2] J. Kijowski, D. Chruściński, Variational Principle for Electrodynamics of Moving Particles, Gen. Rel. and Grav. 27 (1995) 267

[3] C. Misner, K. S. Thorne, J. A. Wheeler Gravitation, W. H. Freeman and Co., San Francisco (1973) 